\title{
Hankel determinant problem of a subclass of analytic functions
}

\author{
Muhammad Arif ${ }^{*}$, Khalida Inayat Noor ${ }^{2}$ and Mohsan Raza²
}

* Correspondence:

marifmaths@yahoo.com

'Department of Mathematics,

Abdul Wali Khan University,

Mardan, Pakistan

Full list of author information is

available at the end of the article

\section{Abstract}

In this article, we study the Hankel determinant problem of a subclass of analytic functions introduced recently by Arif et al.

2010 Mathematics Subject Classification: 30C45; 30C10.

Keywords: Robertson function, strongly Bazilevic functions, bounded boundary rotations, Hankel determinant

\section{Introduction}

Let $\mathcal{A}$ be the class of analytic function satisfying the condition $f(0)=0, f(0)-1=0$ in the open unit $\operatorname{disc} \mathcal{E}=\{z:|z|<1\}$. By $\mathcal{S}, \mathcal{S}^{*}, \mathcal{C}$, and $\mathcal{K}$ we means the well-known subclasses of $\mathcal{A}$ which consist of univalent, starlike, convex, and close-to-convex functions, respectively.

Let $\mathcal{V}_{k}^{\lambda}(\sigma), k \geq 2,0 \leq \sigma<1, \lambda$ real, $|\lambda|<\frac{\pi}{2}$, denote the class of functions $f_{1}(z)$ analytic and locally univalent in $\mathscr{E}, f_{1}(0)=0, f_{1}^{\prime}(0)=1$ and satisfying

$$
\int_{0}^{2 \pi}\left|\left\{\operatorname{Re} e^{i \lambda} \frac{\left(z f_{1}^{\prime}(z)\right)^{\prime}}{f_{1}^{\prime}(z)}-\sigma \cos \lambda\right\} /(1-\sigma)\right| d \theta \leq k \pi \cos \lambda, z=r e^{i \theta} .
$$

This class was introduced and studied in details by Moulis [1]. For $\lambda=0$, we obtain the class $\mathcal{V}_{k}(\sigma)$ of analytic functions with bounded boundary rotations of order $\sigma$ studied by Padmanabhan et al. [2] and when $\sigma=0$ and $\lambda=0$, we get the class $\mathcal{V}_{k}$ discussed by Paatero [3], see also [4-8]. Also it can easily be shown that $f_{1}(z) \in \mathcal{V}_{k}^{\lambda}(\sigma)$ if and only if there exists $f_{2}(z) \in \mathcal{V}_{k}$ such that

$$
f_{1}^{\prime}(z)=\left(f_{2}^{\prime}(z)\right)^{(1-\sigma)^{-i \lambda} \cos \lambda} \text {. }
$$

We now consider a class of analytic functions defined by Arif et al. [9] as follows:

Definition 1.1. Let $f(z) \in \mathcal{A}$ in $E$. Then $f(z) \in \tilde{\mathcal{B}}_{k}(\lambda, \sigma, \beta, \gamma)$, if for $k \geq 2,0 \leq \beta \leq$ $1,0 \leq \gamma \leq 1, \lambda$ is real with $|\lambda|<\frac{\pi}{2}$ there exists a function $f_{1}(z) \in \mathcal{V}_{k}^{\lambda}(\sigma), 0 \leq \sigma<1$, such that

$$
\left|\arg \left\{\frac{z^{1-\gamma} f^{\prime}(z)}{f(z)}\left(\frac{f(z)}{f_{1}^{\prime}(z)}\right)^{\gamma}\right\}\right| \leq \frac{\beta \pi}{2}, z \in E .
$$

(C) 2012 Arif et al; licensee Springer. This is an Open Access article distributed under the terms of the Creative Commons Attribution License (http://creativecommons.org/licenses/by/2.0), which permits unrestricted use, distribution, and reproduction in any medium, provided the original work is properly cited. 
By giving specific values to the parameters $k, \sigma, \lambda, \beta$, and $\gamma$ in $\tilde{\mathcal{B}}_{k}(\lambda, \sigma, \beta, \gamma)$, we obtain many important subclasses studied by various authors in earlier articles, see [10-16].

Using (1.1) and (1.2), we have

$$
z f^{\prime}(z)=z^{\gamma}(f(z))^{1-\gamma}\left(f_{1}^{\prime}(z)\right)^{\gamma} p^{\beta}(z)
$$

where $f_{1}(z) \in \mathcal{V}_{k}^{\lambda}(\sigma)$ and $p(z)$ belongs to the class $\mathcal{P}$ of functions whose real part is positive.

Throughout in this article, we shall assume, unless otherwise stated, that $k \geq 2,0 \leq \beta$ $\leq 1,0<\gamma \leq 1, \lambda$ is real with $|\lambda|<\frac{\pi}{2}, 0 \leq \sigma<1$.

In [17], the $q$ th Hankel determinant $\mathcal{H}_{q}(n), q \geq 1, n \geq 1$, for a function $f(z) \in \mathcal{A}$ is stated by Noonan and Thomas as:

Definition 1.2. Let $f(z) \in \mathcal{A}$. Then the $q$ th Hankel determinant of $f(z)$ is defined for $q \geq 1, n \geq 1$ by

$$
H_{q}(n)=\left|\begin{array}{cccc}
a_{n} & a_{n+1} & \cdots & a_{n+q-1} \\
a_{n+1} & a_{n+2} & \cdots & a_{n+q-2} \\
\vdots & \vdots & \vdots & \vdots \\
a_{n+q-1} & a_{n+q-2} & \cdots & a_{n+2 q-2}
\end{array}\right|
$$

The Hankel determinant plays an important role, for instance, in the study of the singularities by Hadamard, see [[18], p. 329], Edrei [19] and in the study of power series with integral coefficients by Polya [[20], p. 323], Cantor [21], and many others.

In this article, we shall determine the rate of growth of the Hankel determinant $\mathcal{H}_{q}(n)$ for $f(z) \in \tilde{\mathcal{B}}_{k}(\lambda, \sigma, \beta, \gamma)$ with $0<\beta<2$, as $n \rightarrow \infty$. This determinant has been considered by several authors. That is, Noor [22] determined the rate of growth of $\mathcal{H}_{q}(n)$ as $n \rightarrow \infty$ for a function $f(z)$ belongs to the class $\mathcal{V}_{k}$. Pommerenke in [23] studied the Hankel determinant for starlike functions. The Hankel determinant problem for other interesting classes of analytic functions were discussed by Noor [11,12,24].

Lemma 1.1. Let $f(z) \in \mathcal{A}$. Let the $q$ th Hankel determinant of $f(z)$ for $q \geq 1, n \geq 1$ be defined by (1.4). Then, writting $\Delta_{j}(n)=\Delta_{j}\left(n, z_{1}, f(z)\right)$, we have

$$
\mathcal{H}_{q}(n)=\left|\begin{array}{cccc}
\Delta_{2 q-2}(n) & \Delta_{2 q-3}(n+1) & \cdots & \Delta_{q-1}(n+q-1) \\
\Delta_{2 q-3}(n+1) & \Delta_{2 q-4}(n+2) & \cdots & \Delta_{q-2}(n+q-2) \\
\vdots & \vdots & \vdots & \vdots \\
\Delta_{q-1}(n+q-1) & \Delta_{q-2}(n+q-2) & \cdots & \Delta_{q}(n+2 q-2)
\end{array}\right|,
$$

where with $\Delta_{0}(n)=a_{n}$, we define for $j \geq 1$,

$$
\Delta_{j}\left(n, z_{1}, f(z)\right)=\Delta_{j-1}\left(n, z_{1}, f(z)\right)-\Delta_{j-1}\left(n+1, z_{1}, f(z)\right) .
$$

Lemma 1.2. With $z_{1}=\frac{n}{n+1} y$ and $v \geq 0$ any integer,

$$
\Delta_{j}\left(n+v, z_{1}, z f^{\prime}(z)\right)=\sum_{m=0}^{j}\left(\begin{array}{l}
j \\
m
\end{array}\right) \frac{y^{m}(v-(m-1) n)}{(n+1)^{m}} \Delta_{j-m}(n+m+v, f(z)) .
$$

Lemmas 1.1 and 1.2 are due to Noonan and Thomas [17]. 
Lemma 1.3. Let $h_{1}(z)$ be starlike univalent function in $\mathscr{E}$. Then

(i) there exists a $z_{1}$ with $\left|z_{1}\right|=r$ such that for all $z,|z|=r$

$$
\left|z-z_{1}\right|\left|h_{1}(z)\right| \leq \frac{2 r^{2}}{1-r^{2}}
$$

see $[25]$

(ii)

$$
\frac{r}{(1+r)^{2}} \leq\left|h_{1}(z)\right| \leq \frac{r}{(1+r)^{2}},
$$

see[26].

\section{Hankel determinant problem}

Theorem 2.1. Let $f(z) \in \tilde{\mathcal{B}}_{k}(\lambda, \sigma, \beta, \gamma)$ with $0<\beta<2$ and let the $q$ th Hankel determinant $\mathcal{H}_{q}(n)$ of $f(z)$ be defined as in (1.4). Then

$$
\mathcal{H}_{q}(n)=\mathrm{O}(1)(M(r))^{1-\gamma}\left\{\begin{array}{l}
{ }_{n}{ }^{\left(\frac{k}{2}+1\right)(1-\sigma) \cos ^{2} \lambda+\beta-2}, q=1 \\
\left\{\gamma\left(\frac{k}{2}+1\right)(1-\sigma) \cos ^{2} \lambda+\beta-1\right\} \\
\left.{ }_{n}\right\}^{q-q^{2}}, q \geq 2, k \geq \frac{8(q-1)}{(1-\sigma) \gamma \cos ^{2} \lambda}-2,
\end{array}\right.
$$

where $k>\frac{4 j+2-\beta}{(1-\sigma) \gamma \cos ^{2} \lambda}-2$ and $\mathrm{O}(1)$ is a constant depending on $k, \lambda, \beta, \sigma, \gamma$, and $j$ only.

Proof. It is well known [1] that for starlike functions $h_{1}(z)$ and $h_{2}(z)$

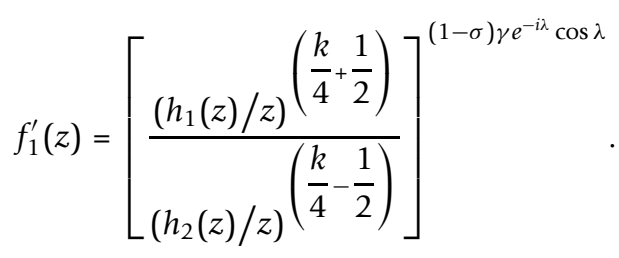

Using (2.1) in (1.3), we have

$$
\left.z f^{\prime}(z)=z^{\gamma}(f(z))^{1-\gamma}\left[\frac{\left(h_{1}(z) / z\right)\left(\frac{k}{4}+\frac{1}{2}\right)}{\left(h_{2}(z) / z\right)}\right]^{\left(\frac{k}{4}-\frac{1}{2}\right)}\right]^{(1-\sigma) \gamma e^{-i \lambda} \cos \lambda} p^{\beta}(z)
$$

where $p(z) \in \mathcal{P}$.

Let $F(z)=z f(z)$. Then for $j \geq 1, z_{1}$ any non-zero complex and $z=r e^{i \theta}$, consider $\Delta_{j}(n$, $\left.z_{1}, F(z)\right)$ as defined by (1.5). Then

$$
\left|\Delta_{j}\left(n, z_{1}, F(z)\right)\right|=\frac{1}{2 \pi r^{n+j}}\left|\int_{0}^{2 \pi}\left(z-z_{1}\right)^{j} F(z) e^{i(n+j) \theta} d \theta\right|,
$$


and by using (2.2), we have

$$
\begin{aligned}
& \left|\Delta_{j}\left(n, z_{1}, F(z)\right)\right| \leq \frac{1}{2 \pi r^{n+j}} \int_{0}^{2 \pi}\left|z-z_{1}\right|^{j}|z|^{\gamma}|f(z)|^{1-\gamma}\left|\left[\frac{\left(h_{1}(z) / z\right)^{\left(\frac{k}{4}+\frac{1}{2}\right)}}{\left(h_{2}(z) / z\right)^{\left(\frac{k}{4}-\frac{1}{2}\right)}}\right]^{(1-\sigma) \gamma e^{-i \lambda} \cos \lambda}\right||p(z)|^{\beta} d \theta \\
& \leq \frac{(M(r))^{1-\gamma}}{2 \pi r^{n+j}} \int_{0}^{2 \pi}\left|z-z_{1}\right|^{j}\left|\left[\frac{\left(h_{1}(z) / z\right)^{\left(\frac{k}{4}+\frac{1}{2}\right)}}{\left(h_{2}(z) / z\right)^{\left(\frac{k}{4}-\frac{1}{2}\right)}}\right]^{(1-\sigma) \gamma e^{-i \lambda} \cos \lambda \mid}\right||p(z)|^{\beta} d \theta .
\end{aligned}
$$

Since

$$
\begin{aligned}
\left|\left[\frac{\left(h_{1}(z) / z\right)^{\left(\frac{k}{4}+\frac{1}{2}\right)}}{\left(h_{2}(z) / z\right)^{\left(\frac{k}{4}-\frac{1}{2}\right)}}\right]^{(1-\sigma) \gamma e^{-i \lambda} \cos \lambda}\right| & \leq\left[\frac{\left|\left(h_{1}(z) / z\right)\right|^{\left(\frac{k}{4}+\frac{1}{2}\right)}}{\left|\left(h_{2}(z) / z\right)\right|\left(\frac{k}{4}-\frac{1}{2}\right)}\right]^{\gamma(1-\sigma) \cos ^{2} \lambda} e^{\gamma(1-\sigma) \gamma \frac{\pi}{2} \frac{\sin 2 \lambda}{2}} \\
& =\frac{\left|\left(h_{1}(z) / z\right)\right|^{\left(\frac{k}{4}+\frac{1}{2}\right) \gamma(1-\sigma) \cos ^{2} \lambda}}{\left|\left(h_{2}(z) / z\right)\right|\left(\frac{k}{4}-\frac{1}{2}\right) \gamma(1-\sigma) \cos ^{2} \lambda} c_{1}, \text { say }
\end{aligned}
$$

therefore (2.3) becomes

$$
\left|\Delta_{j}\left(n, z_{1}, F(z)\right)\right| \leq \frac{c_{1}(M(r))^{1-\gamma}}{2 \pi r^{n+j}} \int_{0}^{2 \pi}\left|z-z_{1}\right|^{j} \frac{\left|\left(h_{1}(z) / z\right)\right|^{\left(\frac{k}{4}+\frac{1}{2}\right) \gamma(1-\sigma) \cos ^{2} \lambda}}{\left|\left(h_{2}(z) / z\right)\right|^{\left(\frac{k}{4}-\frac{1}{2}\right) \gamma(1-\sigma) \cos ^{2} \lambda}}|p(z)|^{\beta} d \theta .
$$

Now using Lemma 1.3, we have

$$
\begin{aligned}
\left|\Delta_{j}\left(n, z_{1}, F(z)\right)\right| & \leq \frac{c_{1}(M(r))^{1-\gamma}}{2 \pi r^{n+j}}\left(\frac{(1+r)^{2}}{r}\right)^{\left(\frac{k}{4}-\frac{1}{2}\right) \gamma(1-\sigma) \cos ^{2} \lambda} \\
& \frac{1}{r^{\gamma(1-\sigma) \cos ^{2} \lambda}}\left(\frac{2 r^{2}}{1-r^{2}}\right)^{j} \int_{0}^{2 \pi}\left|\left(h_{1}(z)\right)\right|^{\left(\frac{k}{4}+\frac{1}{2}\right) \gamma(1-\sigma) \cos ^{2} \lambda-j}|p(z)|^{\beta} d \theta .
\end{aligned}
$$

The well-known Holder's inequality will give us

$$
\begin{aligned}
& \left|\Delta_{j}\left(n, z_{1}, F(z)\right)\right| \leq \frac{c_{1}(M(r))^{1-\gamma} 2^{\left(\frac{k}{4}-\frac{1}{2}\right) \gamma(1-\sigma) \cos ^{2} \lambda+j}}{r^{n-j+\left(\frac{k}{4}+\frac{1}{2}\right) \gamma(1-\sigma) \cos ^{2} \lambda}}\left(\frac{1}{1-r}\right)^{j}\left(\frac{1}{2 \pi} \int_{0}^{2 \pi}|p(z)|^{2} d \theta\right)^{\frac{\beta}{2}} \\
& \left(\frac{1}{2 \pi} \int_{0}^{2 \pi}\left|\left(h_{1}(z)\right)\right| \quad d \theta\right)^{\frac{\left(\frac{k}{2}+1\right) \gamma(1-\sigma) \cos ^{2} \lambda-2 j}{2-\beta}}
\end{aligned}
$$

Also, it is known [15] that, for $p(z) \in \mathcal{P}, z \in E$,

$$
\frac{1}{2 \pi} \int_{0}^{2 \pi}|p(z)|^{2} d \theta \leq \frac{1+3 r^{2}}{1-r^{2}} .
$$


Using (2.5) in (2.4), we obtain

$$
\begin{aligned}
\left|\Delta_{j}\left(n, z_{1}, F(z)\right)\right| \leq \frac{c_{1}(M(r))^{1-\gamma} 2\left(\frac{k}{4}-\frac{1}{2}\right) \gamma(1-\sigma) \cos ^{2} \lambda+j}{r^{n-j+\left(\frac{k}{4}+\frac{1}{2}\right)}\left(\frac{1}{2}(1-\sigma) \cos ^{2} \lambda\right.}\left(\frac{1}{1-r}\right)^{j} \\
\left(\frac{1+3 r^{2}}{1-r^{2}}\right)^{\frac{\beta}{2}}\left(\frac{1}{2 \pi} \int_{0}^{2 \pi}\left|\left(h_{1}(z)\right)\right| \frac{\left(\frac{k}{4}+1\right) \gamma(1-\sigma) \cos ^{2} \lambda-2 j}{2-\beta} d \theta\right)^{\frac{2-\beta}{2}} .
\end{aligned}
$$

Therefore, we can write

$$
\begin{aligned}
& \left|\Delta_{j}\left(n, z_{1}, F(z)\right)\right| \leq \frac{c_{1}(M(r))^{1-\gamma} 2\left(\frac{k}{4}-\frac{1}{2}\right) \gamma(1-\sigma) \cos ^{2} \lambda+\beta+j}{r^{n-j+}\left(\frac{k}{4}+\frac{1}{2}\right) \gamma(1-\sigma) \cos ^{2} \lambda}\left(\frac{1}{1-r}\right)^{j+\frac{\beta}{2}} \\
& {\left[r^{\left(\frac{k}{4}+\frac{1}{2}\right) \gamma(1-\sigma) \cos ^{2} \lambda-j} \frac{1}{2 \pi} \int_{0}^{2 \pi} \frac{d \theta}{\mid 1-r e^{i \theta \mid}}\right]^{\frac{(k+2) \gamma(1-\sigma) \cos ^{2} \lambda-4 j}{2-\beta}} .}
\end{aligned}
$$

Now using a subordination result for starlike functions, we have

$$
\begin{aligned}
\left|\Delta_{j}\left(n, z_{1}, F(z)\right)\right| & \leq \frac{c_{2}(M(r))^{1-\gamma}}{r^{n}}\left(\frac{1}{1-r}\right)^{j+\frac{\beta}{2}}\left[\left(\frac{1}{1-r}\right)^{\frac{\gamma(k+2)(1-\sigma) \cos ^{2} \lambda-4 j}{2-\beta}-1}\right]^{\frac{2-\beta}{2}}, \\
& =\frac{c_{2}(M(r))^{1-\gamma}}{r^{n}}\left(\frac{1}{1-r}\right)^{\gamma\left(\frac{k}{2}+1\right)(1-\sigma) \cos ^{2} \lambda+\beta-1-j},
\end{aligned}
$$

where $c_{2}$ is a constant depending on $k, \lambda, \beta, \sigma, \gamma, j$ only and $\gamma(k+2)(1-\sigma) \cos ^{2} \lambda-$ $4 j>2-\beta$.

Applying Lemma 1.2 and putting $z_{1}=\left(\frac{n}{n+1}\right) e^{i \theta_{n}},(n \rightarrow \infty), r=1-\frac{1}{n}$, we have for

$\left|\Delta_{j}\left(n, e^{i \theta_{n}}, f(z)\right)\right|=\mathrm{O}(1)(M(r))^{1-\gamma} n^{\gamma\left(\frac{k}{2}+1\right)(1-\sigma) \cos ^{2} \lambda+\beta-j-2}$,

$$
\left|\Delta_{j}\left(n, e^{i \theta_{n}}, f(z)\right)\right|=\mathrm{O}(1)(M(r))^{1-\gamma} n^{\gamma\left(\frac{k}{2}+1\right)(1-\sigma) \cos ^{2} \lambda+\beta-j-2},
$$

where $\mathrm{O}(1)$ is a constant depending on $k, \lambda, \beta, \sigma, \gamma$, and $j$ only.

We now estimate the rate of growth of $\mathcal{H}_{q}(n)$.

For $q=1, \mathcal{H}_{q}(n)=a_{n}=\Delta_{0}(n)$ and

$$
H_{1}(n)=a_{n}=\mathrm{O}(1)(M(r))^{1-\gamma} n^{\gamma\left(\frac{k}{2}+1\right)(1-\sigma) \cos ^{2} \lambda+\beta-2} .
$$

For $q \geq 2$, we use similar argument due to Noonan and Thomas [17] together with Lemma 1.1 to have

$$
\mathcal{H}_{q}(n)=\mathrm{O}(1)(M(r))^{1-\gamma_{n}}\left[\gamma\left(\frac{k}{2}+1\right)(1-\sigma) \cos ^{2} \lambda+\beta-1\right] q-q^{2}, k \geq \frac{8(q-1)}{(1-\sigma) \gamma \cos ^{2} \lambda}-2
$$


and $\mathrm{O}(1)$ depends only on $k, \lambda, \beta, \sigma, \gamma$, and $j$.

For choosing different values of $\lambda, \beta, \sigma$, and $\gamma$ in Theorem 2.1, we obtain the following results discussed by Noor $[10,11]$ and Noor et al. [14].

Corollary 2.1. For $\lambda=0, \beta=1, \sigma=0, f(z) \in \mathcal{B}_{k}(\gamma)$, where the class $\mathcal{B}_{k}(\gamma)$ was introduced by Noor et al. [14] and

$$
\mathcal{H}_{q}(n)=\mathrm{O}(1)(M(r))^{1-\gamma}\left\{\begin{array}{l}
n^{\gamma\left(\frac{k}{2}+1\right)-1}, q=1 \\
n^{\gamma\left(\frac{k}{2}+1\right) q-q^{2}}, q \geq 2, k \geq \frac{8(q-1)}{\gamma}-2
\end{array}\right.
$$

where $\mathrm{O}(1)$ is a constant depending only on $k$ and $\gamma$.

Corollary 2.2. For $\lambda=0, \gamma=1, f(z) \in \tilde{\mathcal{T}}_{k}(\beta, \sigma)$ and

$$
\mathcal{H}_{q}(n)=\mathrm{O}(1)\left\{\begin{array}{l}
n^{(1-\sigma)\left(\frac{k}{2}+1\right)+\beta-2}, q=1 \\
\left.n^{\left[(1-\sigma)\left(\frac{k}{2}+1\right)+\beta-1\right]}\right] q-q^{2}, q \geq 2, k \geq \frac{8(q-1)}{(1-\sigma)}-2
\end{array}\right.
$$

where $\mathrm{O}(1)$ is a constant depends on $k, \sigma$, and $\beta$ only.

\section{Author details}

${ }^{1}$ Department of Mathematics, Abdul Wali Khan University, Mardan, Pakistan ${ }^{2}$ COMSATS Institute of Information Technology, Islamabad, Pakistan

\section{Authors' contributions}

MA, KIN, and MR jointly discussed and presented the ideas of this article. MA made the text file and all the communications regarding the manuscript. All authors read and approved the final manuscript.

\section{Competing interests}

The authors declare that they have no competing interests.

Received: 2 October 2011 Accepted: 8 February 2012 Published: 8 February 2012

\section{References}

1. Moulis, EJ: Generalizations of the Robertson functions. Pac J Math. 81(1):167-174 (1979)

2. Padmanabhan, K, Parvatham, R: Properties of a class of functions with bounded boundary rotation. Ann Polon Math. 31, 311-323 (1975)

3. Paatero, V: Uber Gebiete von beschrankter Randdrehung. Ann Acad Sci Fenn Ser A. 37(9):20 (1933)

4. Noor, MA, Noor, KI, Al-Said, EA: On certain analytic functions with bounded radius rotation. Comput Math Appl. 61(10):2987-2993 (2011). doi:10.1016/j.camwa.2011.03.084

5. Noor, Kl, Haq, W, Arif, M, Mustafa, S: On bounded boundary and bounded radius rotations. J Inequal Appl, vol 2009 (2009). Art. ID 813687, 12

6. Noor, Kl, Noor, MA, Al-Said, EA: On multivalent functions of bounded radius rotations. Appl Math Lett. 24(7):1155-1159 (2011). doi:10.1016/j.aml.2011.01.042

7. Noor, KI, Noor, MA, Al-Said, EA: On analytic functions of bounded boundary rotation of complex order. Comput Math Appl. 62, 2112-2125 (2011). doi:10.1016/j.camwa.2011.06.059

8. Arif, M, Noor, Kl, Raza, M: On a class of analytic functions related with generalized Bazilevic type functions. Comput Math Appl. 61, 2456-2462 (2011). doi:10.1016/j.camwa.2011.02.026

9. Arif, M, Raza, M, Noor, Kl, Malik, SN: On strongly Bazilevic functions associated with generalized Robertson functions. Math Comput Model. 54, 1608-1612 (2011). doi:10.1016/j.mcm.2011.04.033

10. Noor, Kl: On certain analytic functions related with strongly close-to-convex functions. Appl Math Comput. 197, 149-157 (2008). doi:10.1016/j.amc.2007.07.039

11. Noor, Kl: On the Hankel determinant of close-to-convex univalent functions. Int J Math Math Sci. 3, 447-481 (1980)

12. Noor, Kl: On the Hankel determinant problem for strongly close-to-convex functions. J Nat Geom. 11, 29-34 (1997)

13. Noor, Kl: Some classes of analytic functions related with Bazilevic functions. Tamkang J Math. 28(3):201-204 (1997)

14. Noor, Kl, Al-Bany, SA: On Bazilevic functions. Int J Math Math Sci. 10(1):79-88 (1987). doi:10.1155/S0161171287000103

15. Pommerenke, C: On close-to-convex analytic functions. Trans Am Math Soc. 114, 176-186 (1965). doi:10.1090/500029947-1965-0174720-4

16. Thomas, DK: On Bazilevic functions. Trans Am Math Soc. 132, 353-361 (1968) 
17. Noonan, JW, Thomas, DK: On the Hankel determinant of areally mean p-valent functions. Proc Lond Math Soc. 25(3):503-524 (1972). doi:10.1112/plms/s3-25.3.503

18. Dienes, P: The Taylor series. Dover, New York (1957)

19. Edrei, A: Sur les determinants recurrents et less singularities d'une fonction donee por son developpement de Taylor. Compos Math. 7, 20-88 (1940)

20. Polya, G, Schoenberg, IJ: Remarks on de la Vallee Poussin means and convex conformal maps of the circle. Pac J Math. 8, 259-334 (1958)

21. Cantor, DG: Power series with integral coefficients. Bull Am Math Soc. 69, 362-366 (1963). doi:10.1090/S0002-9904-196310923-4

22. Noor, Kl: Hankel determinant problem for functions of bounded boundary rotations. Rev Roum Math Pures Appl. 28, 731-739 (1983)

23. Pommerenke, C: On starlike and close-to-convex analytic functions. Proc Lond Math Soc. 13, 290-304 (1963). doi:10.1112/plms/s3-13.1.290

24. Noor, Kl, Al-Naggar, IA: Hankel determinant problem. J Nat Geom. 14(2):133-140 (1998)

25. Golusin, GM: On distortion theorem and coefficients of univalent functions. Math Sb. 19, 183-203 (1946)

26. Polatoglu, Y, Bolcal, M: Some results on the Janowski's starlike functions of complex order. arXiv:math/0007133v1

doi:10.1186/1029-242X-2012-22

Cite this article as: Arif et al: Hankel determinant problem of a subclass of analytic functions. Journal of Inequalities and Applications 2012 2012:22.

\section{Submit your manuscript to a SpringerOpen ${ }^{\circ}$} journal and benefit from:

- Convenient online submission

- Rigorous peer review

- Immediate publication on acceptance

- Open access: articles freely available online

- High visibility within the field

- Retaining the copyright to your article

Submit your next manuscript at $\gg$ springeropen.com 